\title{
Mitteilungen der DIVI
}

Intensivmed 2010 · 47:73

DOI 10.1007/s00390-010-0164-7

๑) Springer Medizin Verlag 2010

Deutsche Interdisziplinäre Vereinigung für Intensiv- und Notfallmedizin (DIVI)

Präsident

Prof. Dr. med. G.W. Sybrecht

\section{Geschäftsstelle der DIVI}

c/o Volker Parvu

Universitätsklinikum des Saarlandes

Klinik für Innere Medizin V

66421 Homburg Saar

Tel. 068411621624

Fax 068411623647

info@divi-org.de

www.divi-org.de

\section{Förderstipendium der DIVI-Stiftung 2011}

\section{Stiftung der Deutschen Interdisziplinären \\ Vereinigung für Intensiv- und Notfallmedizin}

Die DIVI-Stiftung vergibt ein Förderstipendium in Form einer einmaligen finanziellen Zuwendung zur Unterstützung von klinischen und wissenschaftlichen Projekten in der Intensivmedizin und Notfallmedizin.

Die finanzielle Zuwendung des Stipendiums dient als Beitrag für Sachmittel und/oder Aufwendungen zur Implementierung und Realisation von wissenschaftlichen Aktivitäten für Nachwuchswissenschaftler (Alter unter 35 Jahren).

Der/die Bewerber/-in sollte Mitglied der DIVI oder einer medizinisch-wissenschaftlichen Gesellschaft sein, die Intensivmedizin und Notfallmedizin als einen der fachlichen Schwerpunkte ausweist bzw. Mitglied der DIVI ist.

Einzelheiten über die DIVIStiftung, Voraussetzungen zur Bewerbung, Informations- und
Antragsdokumente (Download) sind der Homepage der DIVI unter folgender Internet-Adresse zu entnehmen:

- http://divi-org.de/ (Stiftung, Preise und Stipendien)

- http://www.divi-org.de/ preise/downloads/ foerderstipendium.doc

Die Bewerber werden gebeten, den Antrag bis zum 30. Juni 2011 (Ende der Bewerbungsfrist) dem Vorsitzenden der DIVI-Stiftung unter Angabe des Kennworts „Förderstipendium der DIVIStiftung" einzureichen.

Anschrift des Vorsitzenden der DIVI-Stiftung:

Prof. Dr. med. Dr.h.c. Wolfgang Dick

Carl-Orff-Straße 2 55127 Mainz wfdick@t-online.de 